\title{
PREVALENCIA Y FACTORES ASOCIADOS A LA INTENCIÓN DE VACUNARSE CONTRA LA COVID-19 EN EL PERÚ
}

\author{
Percy Herrera-Añazco (1D)1,2,3,a , Ángela Uyen-Cateriano(1)4,b, Diego Urrunaga-Pastor(D),

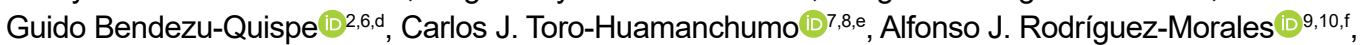 \\ Adrian V. Hernández (D11,12,g, Vicente A. Benites-Zapata(iD) 2,13,h \\ 1 Universidad Privada San Juan Bautista. Lima, Perú. \\ 2 Red Internacional en Salud Colectiva y Salud Intercultural. Ciudad de México, México. \\ 3 Instituto de Evaluación de Tecnologías en Salud e Investigación, EsSalud. Lima, Perú. \\ Médicos Sin Fronteras. Política Sanitaria. Bruselas, Bélgica. \\ ${ }^{5}$ Universidad Científica del Sur. Lima, Perú. \\ ${ }^{6}$ Centro de Investigación Epidemiológica en Salud Global, Universidad Privada Norbert Wiener. Lima, Perú. \\ 7 Facultad de Medicina Humana, Universidad de San Martín de Porres. Chiclayo, Perú. \\ ${ }^{8}$ Unidad de Investigación Multidisciplinaria, Clínica Avendaño. Lima, Perú. \\ ${ }^{9}$ Grupo de Investigación Biomedicina, Facultad de Medicina, Fundación Universitaria Autónoma de las Américas. Pereira, Colombia. \\ ${ }^{10}$ Asociación Colombiana de Infectología. Bogotá, Colombia. \\ ${ }^{11}$ University of Connecticut. Storrs, Connecticut, Estados Unidos. \\ ${ }^{12}$ Unidad de Revisiones Sistemáticas y Meta-análisis, Guías de Práctica Clínica y Evaluaciones Tecnológicas Sanitarias. Universidad San \\ Ignacio de Loyola, Lima, Perú. \\ ${ }^{13}$ Unidad para la Generación y Síntesis de Evidencias en Salud, Universidad San Ignacio de Loyola. Lima, Perú. \\ a Médico nefrólogo, ${ }^{\mathrm{b}}$ magíster en Administración de Empresas de Salud y magíster en Relaciones Internacionales, ${ }^{\mathrm{c}}$ médico cirujano, ${ }^{\mathrm{d}}$ magíster \\ en Informática Biomédica, ${ }^{\mathrm{e}}$ médico epidemiólogo, ${ }^{\mathrm{f}}$ médico tropicalista e investigador sénior; ${ }^{\mathrm{g}}$ doctor en Epidemiología Clínica, ${ }^{\mathrm{h}}$ magíster en \\ Investigación Epidemiológica.
}

\section{RESUMEN}

Objetivos: Estimar la prevalencia y los factores asociados a la intención de vacunación (IDV) contra la COVID-19 en el Perú. Materiales y métodos: Estudio transversal analítico utilizando una encuesta realizada por la Universidad de Maryland, EUA, en Facebook. La variable dependiente fue la IDV. Se calcularon razones de prevalencia (RP) crudas y ajustadas, con sus intervalos de confianza al 95\% (IC95\%), mediante modelos lineales generalizados de la familia Poisson para evaluar la asociación de variables sociodemográficas, el cumplimiento de estrategias comunitarias de mitigación, los síntomas de la COVID-19, la salud mental y la aceptación de la vacunación ante la recomendación (AVR) por diversos actores y autoridades sanitarias. Resultados: Se analizaron los datos de 17162 adultos. La prevalencia general de la IDV fue del 74,9\%. Se asociaron a una menor prevalencia de la IDV ser de sexo femenino (RP = 0,95; IC95\%: 0,94-0,97), vivir en un pueblo ( $R P=0,95$; IC95\%: 0,91-0,99) o en una aldea u otra área rural ( $R P=0,90$; IC95\%: 0,86-0,93) y la AVR de políticos $(\mathrm{RP}=$ 0,89; IC95\%: 0,87-0,92). Contrariamente, tener síntomas de COVID-19 ( $\mathrm{RP}=1,06$; IC95\%: 1,03-1,09), inseguridad económica ( $\mathrm{RP}=1,04$; IC95\%: 1,01-1,06), miedo a enfermar o que un familiar enferme de COVID-19 ( RP = 1,49; IC95\%: 1,36-1,64) y la AVR de familiares y amigos ( $R P=1,10$; IC95\%: 1,08-1,12), trabajadores de la salud ( $R P=1,29$; IC95\%: 1,261,32), la Organización Mundial de la Salud ( $\mathrm{RP}=1,34$; IC95\%: 1,29-1,40) y funcionarios del gobierno ( $\mathrm{RP}=1,18$; IC95\%: 1,15-1,22) se asociaron con mayor prevalencia de IDV. Conclusiones: Tres cuartas partes de los encuestados manifiestan IDV. Existen factores potencialmente modificables que podrían mejorar la aceptación de la vacuna.

Palabras clave: Infecciones por Coronavirus; Betacoronavirus; Vacunación; Negativa a la Vacunación; Perú (fuente: DECS/BIREME).

\section{PREVALENCE AND FACTORS ASSOCIATED WITH THE INTENTION TO BE VACCINATED AGAINST COVID-19 IN PERU}

Citar como: Herrera-Añazco P, Uyen-Cateriano A, Urrunaga-Pastor D, Bendezú-Quispe G, ToroHuamanchumo CJ, Rodríguez-Morales $\mathrm{AJ}$, et al. Prevalencia y factores asociados a la intención de vacunarse contra la COVID19 en el Perú. Rev Peru Med Exp Salud Publica. 2020;38(3):38190. doi: https://doi.org/10.17843/ rpmesp.2021.383.7446

Correspondencia: Vicente A. BenitesZapata, vbeniteszapata@gmail.com

Recibido: 23/02/2021

Aprobado: $14 / 07 / 2021$

En línea: 26/08/2021

\section{ABSTRACT}

Objectives: To estimate the prevalence and factors associated with COVID-19 vaccination intention (VI) in Peru. Materials and methods: Analytical cross-sectional study using the survey conducted by the University of Maryland, USA, on Facebook. The dependent variable is VI. Crude and adjusted prevalence ratios (PR) were calculated, with their $95 \%$ confidence intervals (95\%CI) using generalized linear models of the Poisson family, to evaluate the association of sociodemographic variables, compliance with community mitigation strategies, symptoms of COVID-19, mental health and acceptance of vaccination before the recommendation (AVR) by various actors and health authorities, with the ITV. Results: Data from 17,162 adults were analyzed. The overall prevalence of VI was $74.9 \%$. A lower prevalence of VI was associated with the female sex $(\mathrm{PR}=0.95$; 95\%CI:0.94-0.97), living in a town ( $\mathrm{PR}=0.95$; 95\%CI:0.91-0.99) or village or other rural area (PR=0.90; 95\%CI:0.86-0.93) and the AVR of politicians ( $\mathrm{PR}=0.89$; 95\%CI:0.87-0.92). Conversely, having COVID-19 symptoms ( $\mathrm{PR}=1.06$; 95\%CI:1.03-1.09), economic insecurity ( $\mathrm{PR}=1.04$; 95\%CI:1.01-1.06), fears of becoming seriously ill or that a family member becomes seriously ill from COVID-19 (PR=1.49; 95\%CI:1.36-1.64) and the AVR of family and friends (PR=1.10; 95\%CI: 1.08-1.12), healthcare workers ( $\mathrm{PR}=1.29$; 95\%CI: 1.26-1.32), World Health Organization (PR=1.34; 95\%CI: 1.29-1.40) and government officials $(\mathrm{PR}=1.18$; 95\%CI: 1.15-1.22) was associated with a higher prevalence of VI. Conclusions: Three-quarters of the respondents had VI. There are potentially modifiable factors that could improve vaccine acceptance.

Keywords: COVID-19; SARS-Co-V2; COVID-19 Vaccines; Vaccination; Vaccination Refusal; Peru (source: MeSH NLM). 


\section{INTRODUCCIÓN}

El 7 de junio de 2021, la Organización Mundial de la Salud (OMS) reportó 173005553 casos confirmados de COVID-19, incluidas 3727605 muertes ${ }^{(1)}$. Sin un tratamiento eficaz y con pocas terapias que modifican el curso de la enfermedad, la esperanza global de controlar la enfermedad recae sobre la distribución efectiva y universal de las vacunas disponibles ${ }^{(2)}$.

La vacunación es un punto clave en el éxito del control de enfermedades ${ }^{(3)}$. A pesar del creciente número de vacunas eficaces y seguras en el mercado, la reticencia a las vacunas es un problema creciente de implicancias globales ${ }^{(4)}$. Este fenómeno ha tomado importancia debido a los brotes de enfermedades prevenibles que se encontraban previamente controladas con las vacunas ${ }^{(5)}$. En el contexto de la pandemia, la aceptación de vacunarse contra la COVID-19 es una discusión relevante debido a la desinformación, desconfianza y teorías de la conspiración que han obstaculizado la adopción de otras medidas de mitigación comunitaria contra la enfermedad, en el cual las vacunas no han sido ajenas ${ }^{(6)}$.

Existen diversos estudios relacionados con la aceptación a vacunarse contra la COVID-19. Algunos países como China, Estados Unidos, Ecuador, Malasia, Indonesia, Corea del Sur, Brasil, Sudáfrica, Dinamarca y Reino Unido tienen una alta aceptación en rangos, entre el $65 \%$ y el $97 \%{ }^{(7-10)}$. Por el contrario, otros países tienen una baja aceptación en rangos, entre el 55\% y el 62\%, como Rusia y Francia ${ }^{(11,12)}$. Esta aceptación varía de acuerdo con factores sociodemográficos como el género, pertenecer a minorías étnicas, ser de población rural, los ingresos económicos o factores sociológicos, como las tendencias políticas, entre otros ${ }^{(6,10,13-16)}$. En Latinoamérica y el Caribe, recientemente reportamos que la intención de vacunación hasta febrero de este año fue del $80 \%$, y que los temores de enfermarse gravemente, que un miembro de la familia se enfermara por COVID-19 y tener síntomas depresivos se asociaron con una mayor probabilidad de intención de vacunación ${ }^{(17)}$. Por el contrario, ser de género femenino y no binario se asoció con una menor probabilidad $^{(17)}$.

El Perú ha sido uno de los países más afectados por la pandemia de la COVID-19. Según la sala situacional del Ministerio de Salud (MINSA), para el 7 de junio de 2021, se reportó un acumulado de más de 1,9 millones de casos y más de 186500 fallecidos por la COVID-19 ${ }^{(18)}$. El 7 de febrero de 2021, llegó el primer lote de vacunas al Perú, iniciándose el proceso de vacunación contra la COVID-19. A pesar de que a la fecha se han aplicado más de cuatro millones de dosis ${ }^{(18)}$, al igual que en el resto del mundo, en el Perú, la aceptación de la vacuna no es total. Según una encuesta publicada por Ipsos en febrero de 2021, de existir una vacuna disponible y gratuita contra la COVID-19, el 35\% de la población del país no se vacunaría, siendo la primera razón el miedo a los efec-

\section{MENSAJES CLAVE}

Motivación para realizar el estudio: Pese a que el Perú es uno
de los países más afectados a nivel mundial por la pandemia de
la COVID-19, la prevalencia de la intención de vacunarse ante
esta enfermedad es incierta.

Principales hallazgos: Tres de cada cuatro encuestados en Facebook tienen intención de vacunarse. Existen factores modificables y no modificables asociados a la intención de vacunarse contra la COVID-19 en el Perú.

Implicancias: Estrategias de comunicación dirigidas a grupos poblacionales que influyen en la intención de vacunación pueden favorecer la vacunación contra la COVID-19 en Perú.

tos adversos ${ }^{(19)}$. Un estudio previo realizado por los autores para la intención de vacunarse en Latinoamérica y el Caribe, no incluyó las variaciones en la intención de vacunarse a nivel departamental, de tal forma que se puedan identificar aspectos que puedan individualizar las estrategias de vacunación en cada uno de los departamentos del Perú ${ }^{(17)}$. Por lo expuesto, el objetivo de la presente investigación fue determinar la prevalencia y los factores asociados a la intención de vacunación (IDV) contra la COVID-19 en el Perú.

\section{MATERIALES Y MÉTODOS}

\section{Diseño del estudio y base de datos}

Se realizó un análisis secundario de una base de datos recolectada por la Universidad de Maryland, EUA, y la red social Facebook (Facebook, Inc.) a partir de una encuesta que tuvo como objetivo evaluar distintas características de los encuestados en el contexto de la pandemia por COVID-19. La encuesta incluye información demográfica, autorreporte de sintomatología de COVID-19, evaluación de seguridad alimentaria y económica, salud mental y un módulo sobre actitudes hacia la vacunación. La encuesta se realizó por primera vez el 23 de abril de 2020 y desde entonces fue aplicada diariamente en más de 200 países o territorios, para lo cual se tradujo al idioma principal de cada país ${ }^{(20)}$. La selección de los participantes encuestados fue aleatoria, dentro del marco muestral del total de usuarios de Facebook según región geográfica y país. Asimismo, cada participante seleccionado tuvo un peso de acuerdo con la región y país en el cual hayan respondido la encuesta. En caso de que las personas rechazaran la invitación u omitieran participar en la encuesta, Facebook invitaba a otra persona dentro de la misma zona geográfica que no hubiera respondido la encuesta dentro de las últimas ocho semanas. 


\section{Población y muestra}

La población encuestada incluyó a usuarios de Facebook de 18 años a más. Para este análisis, se incluyó a los participantes del Perú que habían respondido la encuesta entre el 15 de enero y el 1 de febrero ( $n=29140$ adultos). Se excluyó a aquellos participantes de los que no se tenían datos sobre las variables de interés para este estudio. Con ello, se analizaron los datos de 17162 adultos del Perú.

\section{Variables}

El desenlace del estudio fue la IDV. La IDV se evaluó mediante la siguiente pregunta: «Si hoy se le ofreciera una vacuna para prevenir COVID-19, ¿elegiría vacunarse?». Esta pregunta contaba con cuatro posibles respuestas: «Sí, definitivamente», "probablemente sí», "probablemente no», «definitivamente no». Se dicotomizó la variable considerando las dos últimas alternativas como la no IDV contra la COVID-19 y las dos primeras alternativas como la IDV.

\section{Variables independientes}

\section{Características sociodemográficas}

Se incluyó el género (masculino, femenino, no binario), la edad $(18-24,25-34,35-44,45-54,55-64,65-74,75$ a más años) y el área de residencia del participante (ciudad, pueblo, aldea u otra área rural).

Cumplimiento de estrategias comunitarias de mitigación y síntomas de COVID-19 al momento de la encuesta.

Se evaluó la presencia de sintomatología sospechosa de COVID-19 al momento de la encuesta, definida como tres o más de los siguientes síntomas en las últimas 24 horas ${ }^{(21)}$ : fiebre, tos, dificultad respiratoria, cansancio, coriza, dolor muscular, dolor de garganta, dolor de pecho, náuseas, pérdida del olfato, dolor de ojo y dolor de cabeza.

Se incluyó el cumplimiento y adherencia a las tres principales estrategias comunitarias de mitigación para disminuir la transmisión del coronavirus: lavado de manos, uso de mascarilla y distanciamiento físico. Se consideró el cumplimiento del distanciamiento físico cuando el participante reportó no haber estado en contacto directo (incluyendo tocarse, darse las manos, abrazos, besos) durante no más de un minuto en las últimas 24 horas y no haber estado a menos de dos metros de alguna persona con quien no vive actualmente. Se consideró el cumplimiento del lavado de manos cuando los participantes reportaron haberse lavado las manos al menos una vez en las últimas 24 horas. Además, se consideró el cumplimiento del uso de mascarilla cuando los participantes reportaron haber usado una mascarilla en público (al menos en algún momento) durante los últimos siete días. Se creó una variable considerando el cumplimiento de las tres estrategias comunitarias de mitigación.

\section{Salud mental}

Se evaluó el miedo a que el participante o algún miembro de su familia se enferme gravemente de COVID-19 mediante la siguiente pregunta: «¿Qué tan preocupado está de que usted $o$ alguien de su familia inmediata pueda enfermarse gravemente por el coronavirus (COVID-19)?». La pregunta contó con las siguientes posibles respuestas: «Muy preocupado», «algo preocupado», «no muy preocupado», «nada preocupado». Se creó una variable dicotómica considerando la última alternativa como la ausencia de miedo de que el participante o algún miembro de la familia se enferme gravemente de COVID-19 y las tres restantes como la presencia de miedo.

\section{Inseguridad alimentaria y económica}

Se evaluó la seguridad alimentaria mediante la siguiente pregunta: «¿Está preocupado por tener suficientes alimentos para la próxima semana?» Esta pregunta tuvo cuatro posibles respuestas: «Muy preocupado», «algo preocupado», «no muy preocupado», "para nada preocupado». Se dicotomizó la variable considerando las tres primeras alternativas como inseguridad alimentaria.

Se evaluó la seguridad económica mediante la siguiente pregunta: «¿Está preocupado por la economía de su hogar para el próximo mes?». Esta pregunta tuvo cuatro alternativas: «Muy preocupado», «algo preocupado», «no muy preocupado», «para nada preocupado». Se dicotomizó la variable considerando las tres primeras respuestas como inseguridad económica.

Probabilidad de la aceptación de la vacunación ante la recomendación de distintos agentes

Se evaluó la influencia que podrían tener los amigos y familia, los médicos y otros profesionales de la salud que brindan atención médica, la Organización Mundial de Salud (OMS), las autoridades sanitarias gubernamentales y los políticos en la IDV del participante. Esto se evaluó mediante la siguiente pregunta: «¿Sería más o menos probable que se vacunase contra la COVID-19 si se lo recomendara cada uno de los siguientes...?». Esta pregunta tuvo tres respuestas: «Más probable», «más o menos, igual», «menos probable». Se consideraron las dos últimas alternativas como la falta de influencia ante la aceptación de la vacunación, y la primera alternativa como la presencia de influencia ante la aceptación de la vacunación.

\section{Análisis estadístico}

Descargamos la base de datos en formato Microsoft Excel ${ }^{\circledR}$ 2010 y la importamos al paquete estadístico STATA $^{\oplus}$ v14.0 (StataCorp, TX, EUA). Realizamos los análisis estadísticos considerando el muestreo complejo de la encuesta, mediante el comando svy.

Describimos las variables cualitativas empleando frecuencias absolutas y proporciones ponderadas según el muestreo 
complejo de la encuesta con sus respectivos intervalos de confianza al 95\% (IC95\%). Asimismo, realizamos el análisis bivariado entre las covariables de interés y las variables de desenlace mediante la prueba de Chi cuadrado de Pearson con corrección de Rao-Scott, y modelos lineales generalizados de familia Poisson con función de enlace logarítmica para evaluar los factores asociados a la IDV. Calculamos razones de prevalencia crudas $(\mathrm{RPc})$ y ajustadas $(\mathrm{RPa})$ con sus respectivos IC95\%. Empleamos un criterio estadístico para elegir las variables que incluiríamos en el modelo ajustado (aquellas con un $\mathrm{p}<0,05$ en el modelo crudo) y evaluar la posible colinealidad entre las covariables incluidas en el modelo final. La significancia estadística fue fijada en $\mathrm{p}<0,05$.

\section{Aspectos éticos}

Elaboramos el presente estudio usando una base de datos provista por la Universidad de Maryland, sin identificadores personales; por ese motivo, el estudio no requirió la aprobación de un comité institucional de ética. Dado que los participantes dieron su consentimiento antes de iniciar la encuesta, su privacidad no se vio comprometida.

\section{RESULTADOS}

\section{Características de la muestra de estudio}

Se analizó una muestra de 17162 adultos. El 49,8\% $(\mathrm{n}=8512)$ era de género masculino; el $47,1 \%(\mathrm{n}=9124)$ era menor de 35 años, y el $81,2 \%(n=14229)$ vivía en una ciudad. El 29,9\% $(\mathrm{n}=5264)$ tenía sintomatología sospechosa de COVID-19 al momento de la encuesta; el 82,0\% $(n=14026)$ reportó tener inseguridad alimentaria mientras que el 90,2\% $(n=15502)$ refirió inseguridad económica. Además, el 44,6\% ( $\mathrm{n}=7740)$ refirió que tendría mayor aceptación de la vacunación ante una recomendación por parte de autoridades sanitarias gubernamentales, mientras que solo el 8,8\% $(\mathrm{n}=1443)$ refirió mayor aceptación si la recomendación fuera por parte de políticos. El 74,9\% $(\mathrm{n}=13 \mathrm{175})$ tuvo IDV (Tabla 1).

\section{Prevalencia de intención de vacunación según departamentos}

Los departamentos con la prevalencia de IDV más alta fueron Lima provincia (81,4\%), Lima metropolitana $(77,7 \%)$, Junín $(76,7 \%)$, Callao $(75,7 \%)$, Huancavelica $(75,7 \%)$ y Loreto $(75,7 \%)$. Por otro lado, aquellos con la menor IDV fueron Madre de Dios (53,9\%), Ayacucho (66,1\%), Puno (69,5\%), Ucayali $(69,9 \%)$ y Tacna $(70,2 \%)$ (Figura 1$)$.

\section{Análisis bivariado según la intención de vacunación.}

Se hallaron diferencias significativas entre la IDV y las covariables incluidas, a excepción de los grupos de edad $(\mathrm{p}=0,213)$ y el cumplimiento de las estrategias comunitarias de mitigación $(\mathrm{p}=0,062)$ (Tabla 2).

\section{Factores asociados a la intención de vacunación}

En el modelo de regresión ajustado, se asoció el género femenino ( $\mathrm{RPa}=0,95$; IC95\%: 0,95-0,97; $\mathrm{p}<0,001)$, en comparación con el género masculino, a una menor prevalencia de IDV. Asimismo, residir en un pueblo ( $\mathrm{RPa}=0,95$; IC95\%: $0,91-0,99 ; \mathrm{p}=0,034)$, aldea $\mathrm{u}$ otra área rural $(\mathrm{RPa}=0,90$; IC95\%: 0,86-0,93; $p<0,001$ ), en comparación a una ciudad, se asoció a una menor probabilidad de IDV. Además, tener sintomatología de COVID-19 ( $\mathrm{RPa}=1,06$; IC95\%: 1,03$1,09 ; \mathrm{p}<0,001)$, inseguridad económica ( $\mathrm{RPa}=1,04$; IC95\%: $1,01-1,06 ; \mathrm{p}=0,006$ ) y el miedo a enfermarse $\mathrm{o}$ a que algún familiar se enferme de COVID-19 ( $\mathrm{RPa}=1,49$; IC95\%: 1,36$1,64 ; \mathrm{p}<0,001)$ se asoció a una mayor prevalencia de IDV. Por otro lado, las recomendaciones de la OMS $(\mathrm{RPa}=1,34$; IC95\%: 1,29-1,40; $\mathrm{p}<0,001)$, de médicos y otros profesionales de la salud que brindan atención médica $(\mathrm{RPa}=1,29$; IC95\%: 1,26-1,32; $\mathrm{p}<0,001)$, de autoridades sanitarias gubernamentales ( $\mathrm{RPa}=1,18$; IC95\%: 1,15-1,22; $\mathrm{p}<0,001)$ $y$ de familiares y amigos $(\mathrm{RPa}=1,10$; IC95\%: 1,08-1,12; $\mathrm{p}<0,001)$, se asociaron a una mayor prevalencia de IDV. Por el contrario, las recomendaciones por parte de políticos $(\mathrm{RPa}=0,89$; IC95\%: 0,87-0,92; $\mathrm{p}<0,001)$, se asoció a una menor probabilidad de IDV (Tabla 3 ).

\section{DISCUSIÓN}

Nuestros resultados muestran una alta IDV contra la COVID-19. Esto es similar a lo encontrado en un estudio multinacional que incluyó Brasil y que encontró que el $71,5 \%$ de sus participantes informaron tener una muy alta o alguna probabilidad de aceptar la vacuna contra la COVID-19 (11). En este estudio, la aceptabilidad variaba según el país, y llegaba a ser tan alta como $90 \%$ en China o tan baja como $55 \%$ en Rusia ${ }^{(11)}$. Otros estudios en diferentes países también han mostrado diferentes tasas de aceptación ${ }^{(7,14,22)}$. La variabilidad entre estos resultados puede depender de la forma en la que se plantea la pregunta de investigación, lo que limita su comparación ${ }^{(14)}$. Por ejemplo, un estudio multinacional realizado por el Imperial College of London en noviembre del 2020, preguntó si se tenía «definitivamente la intención» de conseguir la vacuna contra la COVID-19, con respuestas que variaron entre el $18 \mathrm{al} 65 \%{ }^{(22)}$. Nuestro estudio no plantea la respuesta en términos de una «intención definitiva», lo que explicaría los menores porcentajes.

La variabilidad en los resultados también puede deberse al momento en el que se realizó el estudio ${ }^{(14)}$. En los Estados Unidos, el porcentaje de aceptación varió del 72\% en abril al $48 \%$ en octubre del $2020^{(14)}$. En Italia, la aceptación de la vacuna incremento después del confinamiento ${ }^{(23)}$. En el Perú, la encuesta de Ipsos mostró que el porcentaje de peruanos que aceptaba ser vacunado disminuyó del $75 \%$ en agosto 
Tabla 1. Análisis descriptivo de las características de la muestra de estudio ( $\mathrm{n}=17$ 162)

\begin{tabular}{|c|c|c|c|}
\hline \multirow{3}{*}{ Características } & \multicolumn{3}{|c|}{ Total } \\
\hline & \multirow{2}{*}{$\begin{array}{c}\begin{array}{c}\text { Frecuencia absoluta de los } \\
\text { participantes incluidos }\end{array} \\
\mathbf{n}\end{array}$} & \multicolumn{2}{|c|}{$\begin{array}{l}\text { Proporción ponderada de cada } \\
\text { categoría }\end{array}$} \\
\hline & & $\%$ & IC95\% \\
\hline \multicolumn{4}{|l|}{ Género } \\
\hline Masculino & 8512 & 49,8 & $47,3-52,4$ \\
\hline Femenino & 8505 & 49,2 & $46,7-51,8$ \\
\hline No binario & 145 & 1,0 & $0,8-1,1$ \\
\hline \multicolumn{4}{|l|}{ Edad (años) } \\
\hline $18-24$ & 4260 & 20,1 & $18,5-21,8$ \\
\hline $25-34$ & 4864 & 27,0 & $26,0-28,0$ \\
\hline $35-44$ & 3625 & 20,9 & $19,7-22,1$ \\
\hline $45-54$ & 2494 & 17,1 & $16,4-17,8$ \\
\hline $55-64$ & 1374 & 9,0 & $8,2-9,8$ \\
\hline $65-74$ & 468 & 5,2 & $4,2-6,4$ \\
\hline 75 o más & 77 & 0,8 & $0,4-1,4$ \\
\hline \multicolumn{4}{|l|}{ Área de residencia } \\
\hline Ciudad & 14229 & 81,2 & $72,0-87,9$ \\
\hline Pueblo & 1756 & 10,8 & $64,8-17,4$ \\
\hline Aldea u otra área rural & 1177 & 8,0 & $5,7-11,1$ \\
\hline \multicolumn{4}{|c|}{ Sintomatología sospechosa de COVID-19 } \\
\hline No & 11898 & 70,1 & $66,0-73,9$ \\
\hline Sí & 5264 & 29,9 & $26,1-34,0$ \\
\hline \multicolumn{4}{|c|}{ Cumplimiento de estrategias comunitarias de mitigación } \\
\hline No & 9120 & 54,1 & $51,2-56,9$ \\
\hline Sí & 8042 & 45,9 & $43,1-48,8$ \\
\hline \multicolumn{4}{|l|}{ Inseguridad alimentaria } \\
\hline No & 3136 & 18,0 & $15,9-20,4$ \\
\hline Sí & 14026 & 82,0 & $80,0-84,1$ \\
\hline \multicolumn{4}{|l|}{ Inseguridad económica } \\
\hline No & 1660 & 9,8 & $9,0-10,6$ \\
\hline Sí & 15502 & 90,2 & $89,4-91,0$ \\
\hline \multicolumn{4}{|c|}{ Miedo porque algún familiar se enferme de COVID-19 } \\
\hline No & 705 & 4,8 & $3,9-6,0$ \\
\hline Sí & 16457 & 95,2 & $94,0-96,1$ \\
\hline \multicolumn{4}{|c|}{ Probabilidad de aceptación de la vacunación ante la recomendación de familiares y amigos } \\
\hline Menor aceptación/Indiferente & 10238 & 60,0 & $58,3-61,7$ \\
\hline Mayor aceptación & 6924 & 40,0 & $38,3-41,7$ \\
\hline \multicolumn{4}{|c|}{$\begin{array}{l}\text { Probabilidad de aceptación de la vacunación ante la recomendación de médicos y otros profesionales de } \\
\text { la salud que brindan atención médica }\end{array}$} \\
\hline Menor aceptación/Indiferente & 8461 & 50,1 & $48,2-52,0$ \\
\hline Mayor aceptación & 8701 & 49,9 & $48,0-51,8$ \\
\hline \multicolumn{4}{|c|}{ Probabilidad de aceptación de la vacunación ante la recomendación de la OMS } \\
\hline Menor aceptación/Indiferente & 7834 & 46,3 & $44,7-47,9$ \\
\hline Mayor aceptación & 9328 & 53,7 & $52,1-55,3$ \\
\hline \multicolumn{4}{|c|}{$\begin{array}{l}\text { Probabilidad de aceptación de la vacunación ante la recomendación de autoridades sanitarias } \\
\text { gubernamentales }\end{array}$} \\
\hline Menor aceptación/Indiferente & 9422 & 55,4 & $53,2-57,5$ \\
\hline Mayor aceptación & 7740 & 44,6 & $42,5-46,8$ \\
\hline \multicolumn{4}{|c|}{ Probabilidad de aceptación de la vacunación ante la recomendación de políticos } \\
\hline Menor aceptación/Indiferente & 15719 & 91,2 & $90,6-91,7$ \\
\hline Mayor aceptación & 1443 & 8,8 & $8,3-9,4$ \\
\hline \multicolumn{4}{|l|}{ Intención de vacunación } \\
\hline No & 3987 & 25,1 & $23,0-27,3$ \\
\hline Sí & 13175 & 74,9 & $72,7-77,0$ \\
\hline \multicolumn{4}{|l|}{ Miedo a efectos adversos de la vacuna } \\
\hline No & 1542 & 9,5 & $8,8-10,2$ \\
\hline Sí & 15620 & 90,5 & $89,8-91,2$ \\
\hline
\end{tabular}

IC95\%: Intervalos de confianza al 95\%. 


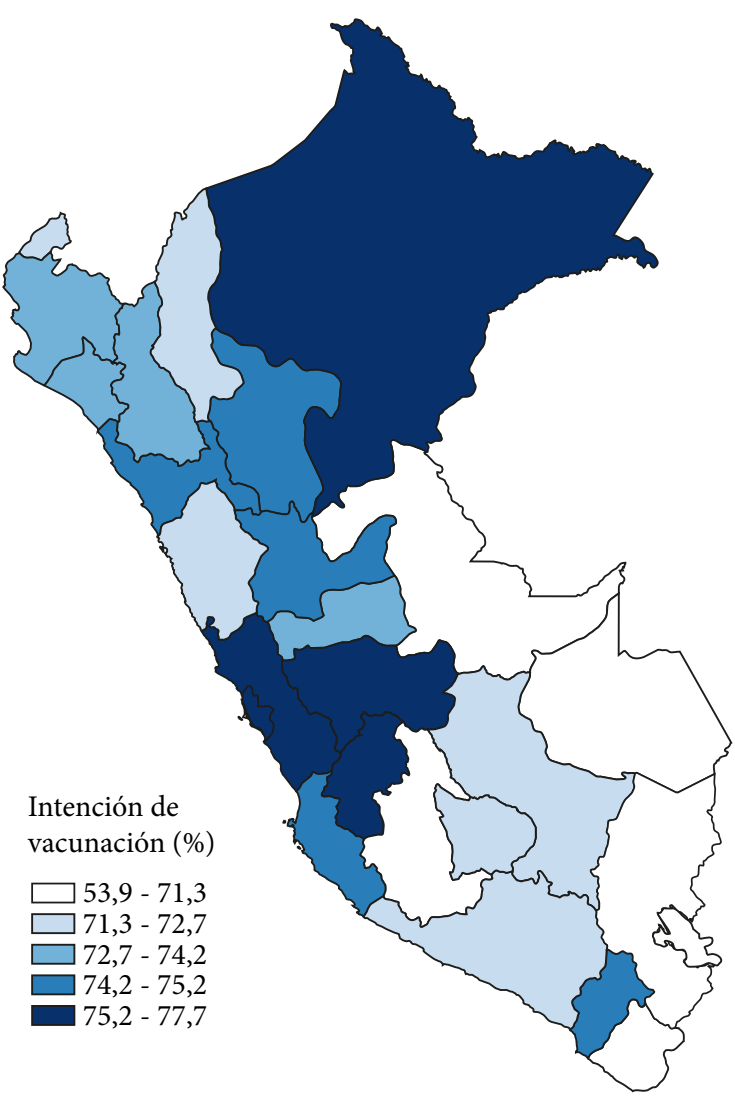

Figura 1. Prevalencia de intención de vacunación según los departamentos del Perú

2020 al 59\% en febrero $2021^{(19)}$. Las diferencias encontradas entre dicha encuesta y nuestro estudio podrían deberse a que la confianza aumentó con el tiempo debido a la llegada de las vacunas y más información acerca de las mismas ${ }^{(24)}$. Un estudio previo en países de la región Latinoamérica y Caribe, que empleó la misma fuente de información de este estudio, reportó que el país con mayor IDV fue México $(88,4 \%)$ y el de menor IDV fue Haití $(43,2 \%)^{(17)}$.

De la misma manera, es posible que conforme la sensación de riesgo en el país aumentó, también lo hizo la IDV ${ }^{(14,23)}$. En semanas previas a la encuesta, debido al aumento del número de casos y fallecidos ${ }^{(18)}$, las noticias de la falta de oxígeno y de camas disponibles en los hospitales, quizá haya aumentado la sensación de vulnerabilidad y, con ella, la aceptación a la vacuna. Un estudio en Turquía mostró que el $40 \%$ de los que inicialmente tuvieron dudas respecto a la vacuna, la consideró necesaria conforme avanzó la pandemia ${ }^{(25)}$. De igual forma, en Francia, más enfermeras cambiaron el rechazo por la vacilación o la aceptación de la vacuna ${ }^{(26)}$.

$\mathrm{Al}$ igual que nuestro estudio, otros países muestran menos IDV en las mujeres ${ }^{(14,27)}$. Aunque las razones de estas diferencias por género no son del todo claras, un estudio multinacional europeo sugirió que podría deberse a que las mujeres mostraban más preocupación respecto a los efectos adversos y a la seguridad de las vacunas que los hombres ${ }^{(27)}$.

Aunque pocos estudios han incluido a población rural ${ }^{(14)}$, algunos aspectos pueden explicar nuestro hallazgo: vivir en una aldea u otra área rural se asoció a una menor IDV. Los residentes de zonas rurales a menudo se muestran reacios a buscar atención médica o a participar en conductas de salud preventiva en comparación con las poblaciones urbanas ${ }^{(28)}$. De igual forma, el difícil acceso a Internet limita la telemedicina ${ }^{(29)}$ y el acceso a información relacionada con la enfermedad y la vacuna, lo que da pie a centralizar la información y su difusión por medios menos rigurosos que promueven terapias sin eficacia en desmedro de la vacuna ${ }^{(30)}$.

$\mathrm{Al}$ igual que nuestro estudio, otros autores han sugerido que el riesgo percibido de infectarse, el miedo a la gravedad de la enfermedad, el antecedente de haberse infectado o conocer a un amigo o familiar infectado fueron predictores de la IDV ${ }^{(14)}$. Estos hallazgos no se limitan a la vacuna contra la COVID-19, pues similares resultados se han encontrado en la aceptabilidad de otras vacunas ${ }^{(31)}$. Por otro lado, la perspectiva de no poder trabajar y con la consiguiente inseguridad económica y problemas de salud mental, podría explicar que tanto las personas con sintomatología depresiva e inseguridad alimentaria estén más propensos a aceptar la vacunación.

La mayoría de estudios sugiere que la influencia del consejo médico es el factor más importante para aceptar la vacunación ${ }^{(14)}$. En nuestro estudio, si bien el consejo de los trabajadores de salud fue significativo, lo fueron más las recomendaciones de la OMS. A pesar de que durante el desarrollo de la pandemia divulgó información discrepante ${ }^{(32)}$, su condición de organismo rector en temas de salud pública le da credibilidad, por lo que reforzar la importancia de sus mensajes aumentaría la IDV en nuestra población. De igual forma, al ser los trabajadores de salud un factor importante, debería considerarse la difusión de mensajes estandarizados basados en evidencia, por ejemplo, durante la teleconsulta o con figuras médicas representativas en las regiones con menos aceptación. En China, la confianza en los médicos como difusores de aspectos relacionados a la vacuna es del $80 \%$ y en Estados Unidos del 62\%. Por otro lado, en los Estados Unidos, solo el 54\% confía en la vacuna si esta cuenta con la aprobación de la Agencia Federal de Medicamentos (FDA) ${ }^{(14)}$. El hecho de que las recomendaciones de políticos estén asociadas a una menor probabilidad de IDV es preocupante, toda vez que su presencia en medios de comunicación es frecuente, sin embargo, es entendible por la falta de confianza en ellos ${ }^{(33)}$. En los Estados Unidos, tanto aspectos políticos como la influencia del expresidente Donald Trump afectaron la aceptación de la vacuna, lo que debería considerarse también en nuestro país ${ }^{(14)}$. 
Tabla 2. Análisis descriptivo y bivariado de las características de estudio según la intención de vacunación en la muestra de estudio ( $\mathrm{n}=17$ 162)

\begin{tabular}{|c|c|c|c|c|c|c|c|}
\hline \multirow{4}{*}{ Características } & \multicolumn{6}{|c|}{ Intención de vacunación } & \multirow{4}{*}{$\begin{array}{l}\text { Valor } \\
\text { de p }\end{array}$} \\
\hline & \multirow{3}{*}{$\begin{array}{c}\begin{array}{c}\text { Frecuencia absoluta } \\
\text { de los participantes } \\
\text { incluidos }\end{array} \\
n \\
\end{array}$} & \multicolumn{2}{|l|}{ No } & \multicolumn{3}{|c|}{ Sí } & \\
\hline & & \multicolumn{2}{|c|}{$\begin{array}{l}\text { Proporción ponderada de } \\
\text { acuerdo con cada categoría }\end{array}$} & \multirow{2}{*}{$\begin{array}{c}\begin{array}{c}\text { Frecuencia absoluta } \\
\text { de los participantes } \\
\text { incluidos }\end{array} \\
\mathbf{n}\end{array}$} & \multicolumn{2}{|c|}{$\begin{array}{l}\text { Proporción } \\
\text { ponderada de acuerdo } \\
\text { con cada categoría }\end{array}$} & \\
\hline & & $\%$ & IC95\% & & $\%$ & IC95\% & \\
\hline \multicolumn{8}{|l|}{ Género } \\
\hline Masculino & 1845 & 23,6 & $23,2-26,3$ & 6667 & 76,4 & $73,7-78,8$ & 0,010 \\
\hline Femenino & 2091 & 26,3 & $24,5-28,3$ & 6414 & 73,7 & $71,7-75,5$ & \\
\hline No binario & 51 & 37,3 & $23,0-54,1$ & 94 & 62,7 & $45,9-77,0$ & \\
\hline \multicolumn{8}{|l|}{ Edad (años) } \\
\hline $18-24$ & 971 & 24,4 & $22,1-26,8$ & 3289 & 75,6 & $73,2-77,9$ & 0,213 \\
\hline $25-34$ & 1110 & 25,4 & $22,6-28,5$ & 3754 & 74,5 & $71,5-77,4$ & \\
\hline $35-44$ & 869 & 25,6 & $23,3-28,1$ & 2756 & 74,4 & $71,9-76,7$ & \\
\hline $45-54$ & 589 & 25,1 & $22,3-28,1$ & 1905 & 74,9 & $71,9-77,7$ & \\
\hline $55-64$ & 323 & 25,1 & $22,1-28,3$ & 1051 & 74,9 & $71,7-77,9$ & \\
\hline $65-74$ & 99 & 22,4 & $18,4-27,1$ & 369 & 77,6 & $72,9-81,6$ & \\
\hline 75 o más & 26 & 35,6 & $25,6-46,9$ & 51 & 64,4 & $53,1-74,4$ & \\
\hline \multicolumn{8}{|l|}{ Área de residencia } \\
\hline Ciudad & 3146 & 23,7 & $22,0-25,6$ & 11083 & 76,3 & $74,4-78,0$ & $<0,001$ \\
\hline Pueblo & 451 & 28,3 & $25,1-31,7$ & 1305 & 71,7 & $68,3-74,9$ & \\
\hline Aldea u otra área rural & 390 & 34,6 & $31,2-38,2$ & 787 & 65,4 & $61,8-68,8$ & \\
\hline \multicolumn{8}{|c|}{ Sintomatología sospechosa de COVID-19 } \\
\hline No & 2861 & 26,6 & $23,4-29,9$ & 9037 & 73,4 & $70,1-76,6$ & 0,007 \\
\hline Sí & 1126 & 21,6 & $20,2-23,1$ & 4138 & 78,4 & $76,9-79,8$ & \\
\hline \multicolumn{8}{|c|}{ Cumplimiento de estrategias comunitarias de mitigación } \\
\hline No & 2034 & 24,4 & $22,3-26,7$ & 7086 & 75,6 & $73,3-77,7$ & 0,062 \\
\hline Sí & 1953 & 25,9 & $23,7-28,2$ & 6089 & 74,1 & $71,8-76,3$ & \\
\hline \multicolumn{8}{|l|}{ Inseguridad alimentaria } \\
\hline No & 800 & 28,9 & $25,3-32,7$ & 2336 & 71,1 & $67,3-74,7$ & 0,001 \\
\hline Sí & 3187 & 24,3 & $22,2-26,4$ & 10839 & 75,7 & $73,6-77,8$ & \\
\hline \multicolumn{8}{|l|}{ Inseguridad económica } \\
\hline No & 463 & 31,0 & $27,3-35,1$ & 1197 & 69,0 & $64,9-72,7$ & $<0,001$ \\
\hline Sí & 3524 & 24,4 & $22,4-26,6$ & 11978 & 75,6 & $73,4-77,6$ & \\
\hline \multicolumn{8}{|c|}{ Miedo porque algún familiar se enferme de COVID-19 } \\
\hline No & 383 & 56,6 & $61,7-61,4$ & 322 & 43,4 & $38,6-48,3$ & $<0,001$ \\
\hline Sí & 3604 & 23,5 & $21,6-25,5$ & 12853 & 76,5 & $74,5-78,4$ & \\
\hline \multicolumn{8}{|c|}{$\begin{array}{l}\text { Probabilidad de aceptación de la vacunación ante la } \\
\text { recomendación de familiares y amigos }\end{array}$} \\
\hline Menor aceptación/Indiferente & 3160 & 33,3 & $30,9-35,8$ & 7078 & 66,7 & $64,2-69,1$ & $<0,001$ \\
\hline Mayor aceptación & 827 & 12,8 & $11,3-14,4$ & 6097 & 87,2 & $85,6-88,7$ & \\
\hline \multicolumn{8}{|c|}{$\begin{array}{l}\text { Probabilidad de aceptación de la vacunación ante la } \\
\text { recomendación de médicos y otros profesionales de la } \\
\text { salud que brindan atención médica }\end{array}$} \\
\hline Menor aceptación/Indiferente & 3431 & 43,3 & $41,0-45,6$ & 5030 & 56,7 & $54,4-59,0$ & $<0,001$ \\
\hline Mayor aceptación & 556 & 6,9 & $5,8-8,1$ & 8145 & 93,1 & $91,9-94,2$ & \\
\hline \multicolumn{8}{|c|}{$\begin{array}{l}\text { Probabilidad de aceptación de la vacunación ante la } \\
\text { recomendación de la OMS }\end{array}$} \\
\hline Menor aceptación/Indiferente & 3249 & 44,4 & $41,7-47,2$ & 4585 & 55,6 & $52,8-58,3$ & $<0,001$ \\
\hline Mayor aceptación & 738 & 8,4 & $7,3-9,7$ & 8590 & 91,6 & $90,3-92,7$ & \\
\hline $\begin{array}{l}\text { Probabilidad de aceptación de la vacu } \\
\text { recomendación de autoridades sanitar }\end{array}$ & & & & & & & \\
\hline Menor aceptación/Indiferente & 3585 & 40,7 & $39,1-42,3$ & 5837 & 59,3 & $57,7-60,9$ & $<0,001$ \\
\hline Mayor aceptación & 402 & 5,8 & $4,4-7,6$ & 7338 & 94,2 & $92,4-95,6$ & \\
\hline $\begin{array}{l}\text { Probabilidad de aceptación de la va } \\
\text { recomendación de políticos }\end{array}$ & & & & & & & \\
\hline Menor aceptación/Indiferente & 3855 & 26,5 & $24,4-28,7$ & 11864 & 73,5 & $71,3-75,6$ & $<0,001$ \\
\hline Mayor aceptación & 132 & 10,5 & $8,0-13,6$ & 1311 & 89,5 & $86,4-92,0$ & \\
\hline
\end{tabular}

IC95\%: Intervalos de confianza al 95\%. 
Tabla 3. Modelos crudos y ajustados de regresión para evaluar la asociación entre las características de estudio y la intención de vacunación en la muestra de estudio

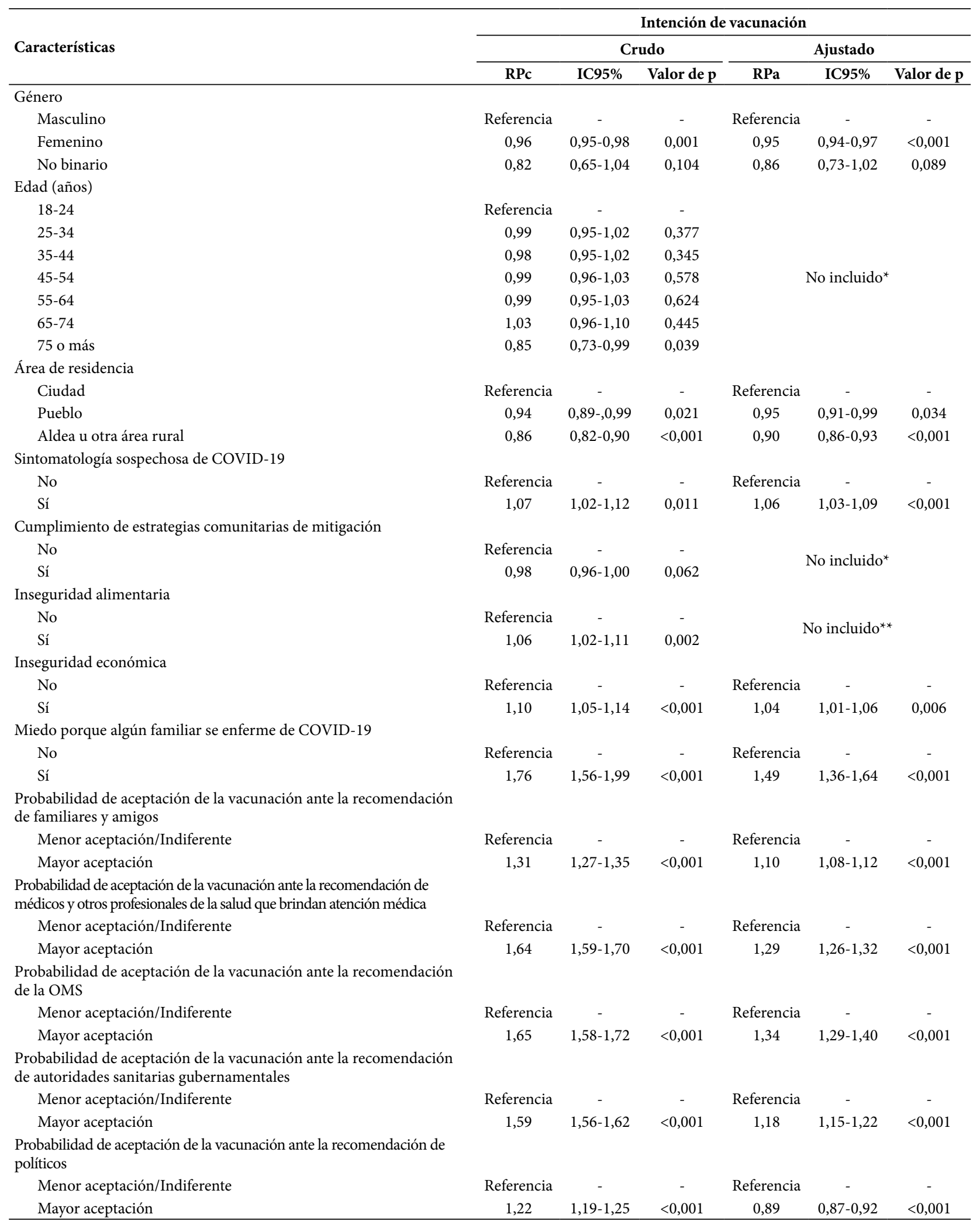

IC95\%: Intervalos de confianza al 95\%; RPc: razón de prevalencia cruda; RPa: razón de prevalencia ajustada. No incluido por no tener asociación estadísticamente significativa en el modelo crudo. ${ }^{* *}$ No incluido por presentar colinealidad con la inseguridad económica. 
Nuestro estudio tiene algunas limitaciones. En primer lugar, está basado en las respuestas de los usuarios de una red social a la que no todos tienen acceso. No obstante, es una red social usada por el $94 \%$ de los peruanos, según una encuesta hecha por Ipsos en el $2020^{(34)}$. En segundo lugar, las variables incluidas y su definición están supeditados a la preestablecida de la encuesta matriz. En tercer lugar, los datos fueron obtenidos por autorreporte, por lo que puede haber un subregistro de información. En cuarto lugar, no se puede establecer causalidad entre las variables evaluadas debido al diseño del estudio. En quinto lugar, las mediciones empleadas para evaluar seguridad alimentaria y económica no han sido validadas, no obstante, permiten brindar información pertinente para el estudio. En sexto lugar, los hallazgos podrían estar sesgados debido a la tasa de rechazo de los usuarios en una encuesta en Facebook, así como debido a la posibilidad de ocurrencia del sesgo de respuesta voluntaria. En séptimo lugar, algunos de los factores asociados hallados tuvieron una medida de asociación baja, por lo cual su interpretación debe de realizarse cautelosamente, pese a la significancia estadística que pueda tener el resultado. A pesar de esto, es un estudio con una

\section{REFERENCIAS BIBLIOGRÁFICAS}

1. WHO. WHOCoronavirus Disease (COVID-19) Dashboard [Internet]. 2021 [citado el 12 de febrero de 2021]. Disponible en: https://covid19.who.int/.

2. Izda V, Jeffries MA, Sawalha AH. COVID-19: A review of therapeutic strategies and vaccine candidates. Clin Immunol. 2020:108634. doi: 10.1016/j.clim.2020.108634.

3. Bach AT, Kang AY, Lewis J, Xavioer S, Portillo I, Goad JA. Addressing common barriers in adult immunizations: a review of interventions. Expert Rev Vaccines. 2019;18(11):1167-85. doi: 10.1080/14760584.2019.1698955.

4. de Figueiredo A, Simas C, Karafillakis E, Paterson P, Larson HJ. Mapping global trends in vaccine confidence and investigating barriers to vaccine uptake: a large-scale retrospective temporal modelling study. Lancet. 2020;396(10255):898-908. doi: 10.1016/S0140-6736(20)31558-0.

5. Kubin $L$. Is there a resurgence of vaccine preventable diseases in the US?. J Pediatr Nurs. 2019;44:115-8. doi: 10.1016/j.pedn.2018.11.011.

6. World Health Organization [Internet]. Behavioural considerations for acceptance and uptake of COVID-19 vaccines: WHO technical advisory group on behavioural insights and sciences for health, meeting report, 15 October 2020. 2020 [citado el 12 de febrero de 2021]. Disponible en: https://www.who.int/publications/i/item/9789240016927.

7. Wang J, Jing R, Lai X, Zhang H, Lyu Y, Knoll MD, et al. Acceptance of COVID-19 Vaccination during the COVID-19 Pandemic in China. Vaccines. 2020;8(3):482. doi: 10.3390/vaccines8030482.

8. Sallam M. COVID-19 vaccine hesitancy worldwide: a concise systematic review of vaccine acceptance rates. Vaccines. 2021;9(2):160. doi: $10.3390 /$ vaccines 9020160 .

9. Sonawane K, Troisi CL, Deshmukh AA. COVID-19 vaccination in the UK: Addressing vaccine hesitancy. Lancet Reg Health Eur. 2021;1. doi: 10.1016/j.lanepe.2020.100016.

10. Pogue K, Jensen JL, Stancil CK, Ferguson DG, Hughes SJ, Mello EJ, et al. Influences on attitudes regarding potential COVID-19 vaccination in the United States. Vaccines. 2020;8(4):582. doi: 10.3390/ vaccines 8040582 . muestra de gran tamaño con representatividad nacional que puede ayudar a entender el tema de estudio.

En conclusión, tres de cada cuatro encuestados manifiestan IDV. Existen factores potencialmente modificables cuya intervención mediante estrategias adecuadas de comunicación aumentaría la posibilidad de aceptación de la vacuna contra la COVID-19 en nuestro país. Considerando que la campaña de vacunación será una tarea de largo plazo, es necesario el monitoreo continuo de la aceptación de la vacuna para enrumbar las estrategias con el fin de conseguir los resultados propuestos por el gobierno.

Conflictos de interés: Los autores declaran no tener conflictos de interés.

Financiamiento: El estudio ha sido autofinanciado.

Agradecimientos: Los autores agradecen a la Universidad de Maryland por la realización de la encuesta con la cual se ha llevado a cabo este trabajo.

Contribuciones de los autores: PHA, DUP y VABZ concibieron el artículo. DUP y VABZ recolectaron y analizaron los datos. PHA, AUC, DUP, GBQ, CJTH, AJRM, AVH y VABZ redactaron el manuscrito y aprobaron su versión final.
11. Lazarus JV, Ratzan SC, Palayew A, Gostin LO, Larson HJ, Rabin K, et al. A global survey of potential acceptance of a COVID-19 vaccine. Nat Med. 2021;27(2):225-8. doi: 0.1038/s41591-020-1124-9.

12. Schwarzinger M, Watson V, Arwidson P, Alla F, Luchini S. COVID-19 vaccine hesitancy in a representative working-age population in France: a survey experiment based on vaccine characteristics. Lancet Public Health. 2021;6(4):e210-e21. doi: 10.1016/S2468-2667(21)00012-8.

13. Malik AA, McFadden SM, Elharake J, Omer SB. Determinants of COVID-19 vaccine acceptance in the US. EClinicalMedicine. 2020;26:100495. doi: 10.1016/j.eclinm.2020.100495.

14. Lin C, Tu P, Beitsch LM. Confidence and Receptivity for COVID-19 Vaccines: A Rapid Systematic Review. Vaccines. 2021;9(1):16. doi: 10.3390/vaccines9010016.

15. Paul E, Steptoe A, Fancourt D. Attitudes towards vaccines and intention to vaccinate against COVID-19: Implications for public health communications. Lancet Reg Health Eur. 2021;1:100012. doi: 10.1016/j. lanepe.2020.100012.

16. Ward JK, Alleaume C, Peretti-Watel P, Seror V, Cortaredona S, Launay $\mathrm{O}$, et al. The French public's attitudes to a future COVID-19 vaccine: The politicization of a public health issue. Soc Sci Med. 2020;265:113414. doi: 10.1016/j.socscimed.2020.113414.

17. Urrunaga-Pastor D, Bendezu-Quispe G, Herrera-Añazco P, Uyen-Cateriano A, Toro-Huamanchumo CJ, Rodriguez-Morales AJ, et al. Cross-sectional analysis of COVID-19 vaccine intention, perceptions and hesitancy across Latin America and the Caribbean. Travel Med Infect Dis. 2021;41:102059. doi: 10.1016/j.tmaid.2021.102059.

18. Ministerio de Salud del Perú [Internet]. Sala Situacional COVID-19 Perú: MINSA Lima; 2020 [citado el 12 de febrero 2021]. Disponible en: https://covid19.minsa.gob.pe/sala_situacional.asp.

19. IPSOS [Internet]. COVID-19 y vacunas- Febrero 2021- Encuesta Nacional Urbano Rural. 2021 [citado el 12 de febrero 2021]. Disponible en: https:/www.ipsos.com/sites/default/files/ct/news/documents/2021-02/ encuesta_nacional_urbano_rural_febrero_2021_-_covid_19.pdf. 
20. Barkay N, Cobb C, Eilat R, Galili T, Haimovich D, LaRocca S, et al. Weights and Methodology Brief for the COVID-19 Symptom Survey by University of Maryland and Carnegie Mellon University, in Partnership with Facebook. arXiv preprint arXiv:200914675. 2020.

21. World Health Organization [Internet]. WHO COVID-19: Case definition. 2020 [citado el 12 de febrero 2021]. Disponible en: https:// www.who.int/publications/i/item/WHO-2019-nCoV-Surveillance_Case_Definition-2020.2.

22. Imperial College London [Internet]. Covid-19: Global attitudes towards a COVID-19 vaccine. 2021 [citado el 12 de febrero 2021]. Disponible en: https://www.imperial.ac.uk/media/imperial-college/ institute-of-global-health-innovation/GlobalVaccineInsights_ ICL-YouGov-Covid-19-Behaviour-Tracker_20210520_v2.pdf

23. Caserotti M, Girardi P, Rubaltelli E, Tasso A, Lotto L, Gavaruzzi T. Associations of COVID-19 risk perception with vaccine hesitancy over time for Italian residents. Soc Sci Med. 2021:113688. doi: 10.1016/j. socscimed.2021.113688.

24. SAGE [Internet]. WHO Strategic Advisory Group of Experts (SAGE) on Immunization Working Group on COVID-19 Vaccines: Prioritized Infectious Disease and Economic Modelling Questions. 2020 [citado el 12 de febrero 2021]. Disponible en: https://www.who.int/ immunization/policy/sage/SAGE_WG_COVID19_Vaccines_Modelling_Questions_31July2020.pdf.

25. Yllmazbas P, Terzi O, Ozceker D. Did. Covid-19 Pandemic Changed Parents' Approach To Vaccination?. Soc Behav. 2021;43(2):130-134. doi: 10.14744/etd.2020.85451.

26. Wang K, Wong ELY, Ho KF, Cheung AWL, Chan EYY, Yeoh EK, et al. Intention of nurses to accept coronavirus disease 2019 vaccination and change of intention to accept seasonal influenza vaccination during the coronavirus disease 2019 pandemic: A cross-sectional survey. Vaccine. 2020;38(45):7049-56. doi: 10.1016/j.vaccine.2020.09.021.
27. Neumann-Böhme S, Varghese NE, Sabat I, Barros PP, Brouwer W, van Exel J, et al. Once we have it, will we use it? A European survey on willingness to be vaccinated against COVID-19. Eur J Health Econ. 2020. doi: 10.1007/s10198-020-01208-6.

28. Peters DJ. Community susceptibility and resiliency to COVID-19 across the rural-urban continuum in the United States. J Rural Health. 2020;36(3):446-56. doi: 10.1111/jrh.12477.

29. Instituto Nacional de Estadística e Informática [Internet]. Estadística de las tecnologías de información y comunicación de los hogares. 2020 [citado el 12 de febrero 2021]. Disponible en: https://www.inei.gob. pe/media/MenuRecursivo/boletines/boletin_tics.pdf.

30. Espacio y análisis [Internet]. El $90 \%$ de muertes asociadas a Covid-19 se encuentran en zonas urbanas. 2021 [citado el 12 de febrero 2021]. Disponible en: https://ojo-publico.com/2003/covid-19-en-regiones90-de-muertes-esta-en-zonas-urbanas.

31. Schmid P, Rauber D, Betsch C, Lidolt G, Denker M-L. Barriers of influenza vaccination intention and behavior-a systematic review of influenza vaccine hesitancy, 2005-2016. PloS One. 2017;12(1):e0170550. doi: 10.1371/journal.pone.0170550.

32. Soave I [Internet]. Alertas, retrasos, cambios, modelos... Los errores de la OMS en la crisis del coronavirus 2020 [citado el 12 de febrero 2021]. Disponible en: https://www.elmundo.es/ciencia-y-salud/salud/2020/05/02/5ead266e21efa01b6b8b464f.html

33. IPSOS [Internet]. Opinión Data - Enero 2021. 2021 [citado el 12 de febrero 2021]. Disponible en: https://www.ipsos.com/es-pe/opinion-data-enero-2021.

34. IPSOS [Internet]. Uso de Redes Sociales entre peruanos conectados. 2020 [citado el 12 de febrero 2021]. Disponible en: https://www.ipsos. com/es-pe/uso-de-redes-sociales-entre-peruanos-conectados-2020. 